\title{
Metallicity and radial velocity of the second parameter globular cluster Terzan 1 in the galactic bulge
}

\author{
T. P. Idiart ${ }^{1}$, B. Barbuy ${ }^{1}$, M.-N. Perrin ${ }^{2}$, S. Ortolani ${ }^{3}$, E. Bica ${ }^{4}$, and A. Renzini ${ }^{5}$ \\ 1 Universidade de São Paulo, CP 3386, São Paulo 04301-904, Brazil \\ e-mail: barbuy@iagusp.usp.br \\ 2 Observatoire de Paris, 61 Av. de l'Observatoire, 75014 Paris, France \\ e-mail: Marie-Noel.Perrin@obspm.fr \\ 3 Università di Padova, Dept. di Astronomia, Vicolo dell'Osservatorio 5, 35122 Padova, Italy \\ e-mail: ortolani@pd.astro.it \\ 4 Universidade Federal do Rio Grande do Sul, Dept. de Astronomia, CP 15051, Porto Alegre 91501-970, Brazil \\ e-mail: bica@if.ufrgs.br \\ 5 European Southern Observatory, Karl Schwarzschild Strasse 2, 85748, Garching bei München, Germany \\ e-mail: arenzini@eso.org
}

Received 21 August 2001 / Accepted 19 October 2001

\begin{abstract}
Terzan 1 is the globular cluster with closest projection relative to the Galactic center. We report radial velocities and metallicities of 17 stars in order to investigate their membership in the cluster. From the 11 probable members of Terzan 1 , a radial velocity of $v_{\mathrm{r}}=114 \pm 14 \mathrm{~km} \mathrm{~s}^{-1}$ is obtained. For this sample an intermediate metallicity of $[\mathrm{Fe} / \mathrm{H}] \approx-1.3$ is found, in agreement with previous work based on HST ColourMagnitude Diagrams.
\end{abstract}

Key words. globular clusters: individual: Terzan 1 - techniques: spectroscopic

\section{Introduction}

The mean age and metallicity of the bulge stellar populations can provide important constraints on the formation and chemical evolution of the Galaxy. Globular clusters located within a few degrees of the Galactic center are strong candidates for membership in the Galactic bulge (Minniti 1995; Barbuy et al. 1998, 1999; Côté 1999). Terzan 1, also named HP 2, has the closest projection to the Galactic center among known globular clusters, with coordinates $\left(\alpha_{1950}=17^{\mathrm{h}} 32^{\mathrm{m}} 34^{\mathrm{s}}, \delta_{1950}=-30^{\circ} 27^{\prime} 0^{\prime \prime}\right.$ and $l=357.56^{\circ}$, $\left.b=0.99^{\circ}\right)$.

The Colour-Magnitude Diagram (CMD) of Terzan 1 has a peculiar morphology, showing a red horizontal branch (HB), which is typical of metal-rich clusters, combined with a very steep red giant branch (RGB), typical in turn of metal-poor clusters. Also, there seems to be a cool

Send offprint requests to: T. P. Idiart,

e-mail: thais@iagusp.usp.br

* Observations collected at the European Southern Observatory, ESO, Chile, Laboratório Nacional de Astrofísica, LNA, Brazil and with the Hubble Space Telescope.
RGB extension curving down to $V \approx 22$ at $(V-I) \approx 5$, which may or may not belong to the cluster population. The combination of the steep RGB and the red HB make it similar to the second parameter halo cluster NGC 362 and Ortolani et al. (1999) concluded that Terzan 1 is a second parameter cluster in the Galactic bulge, and the first one in the bulge combining a steep RGB with a red HB. As in the case of NGC 362, these features of the CMD can be accounted for by the cluster being $~ 2$ Gyr younger than other clusters with the same metallicity but showing a blue horizontal branch. A mean reddening of $E(B-V)=2.48 \pm 0.1($ or $E(V-I)=2.85 \pm 0.1)$ and a distance of $d_{\odot}=5.2 \pm 0.5 \mathrm{kpc}$ were derived (Ortolani et al. 1999).

Therefore, one of the problems to be tackled in the case of Terzan 1 is to understand the extended cool giant sequence combined with the steep RGB. In this work, intermediate resolution spectra of stars in these two CMD sequences of Terzan 1 are obtained, aiming to investigate the membership of each of these individual stars.

In Sect. 2 the observations and reductions are reported. In Sect. 3 estimated kinematical and atmospheric stellar 


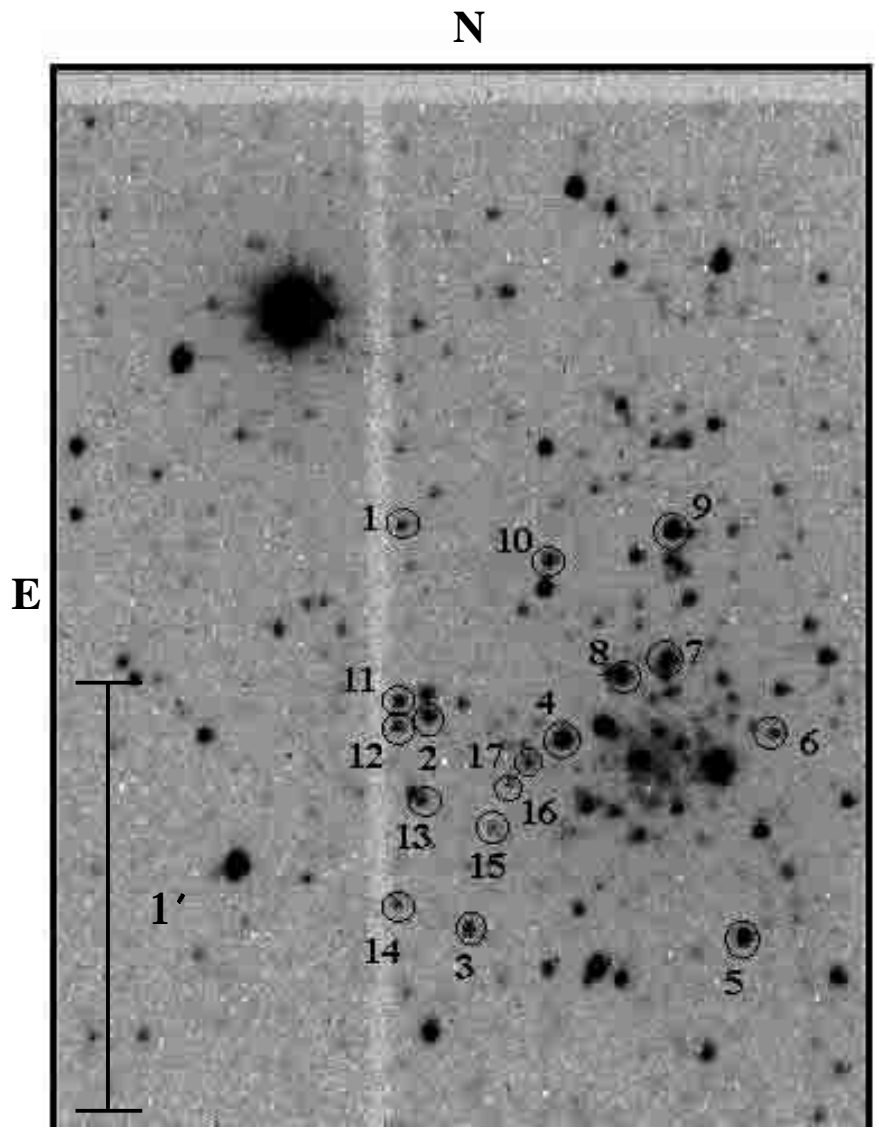

Fig. 1. Image of the globular cluster Terzan 1, obtained with the guiding camera of the LNA $1.6 \mathrm{~m}$ telescope. Dimensions are $2.5^{\prime} \times 2^{\prime}$. Observed stars are marked.

parameters are presented. The results are discussed in Sect. 4 and concluding remarks are drawn in Sect. 5 .

\section{Observations and reductions}

We obtained intermediate resolution spectra for a sample of 17 stars, selected from the CMD of Terzan 1 for being close to the RGB tip. Their location in the CCD frame and in the $V$ vs. $V-I$ CMD are shown in Figs. 1 and 2 respectively. $V$ and $I$ magnitudes were obtained with the Hubble Space Telescope (HST) (Ortolani et al. 1999) for eleven stars, whereas for six others (stars 2, 4, 5, 7, 8 and 9), magnitudes were obtained with the $1.50 \mathrm{~m}$ Danish telescope at the European Southern Observatory - ESO (Ortolani et al. 1993). Corrections of magnitude scale from the Danish to the HST photometry system were carried out using three stars in common (stars 3,6 and 10). We have adopted the HST photometry because the zero point of the Danish data was affected by crowding in the transfer from the uncrowded standard stars to the cluster stars.

The program stars were observed at the Laboratório Nacional de Astrofísica (LNA), Brazil and ESO, Chile. The instrumental configuration is summarized in Table 1 . The spectral coverage is $\lambda \lambda 4800-6600 \AA$ and $\lambda \lambda 3980-7848 \AA$ for the LNA and ESO data respectively.

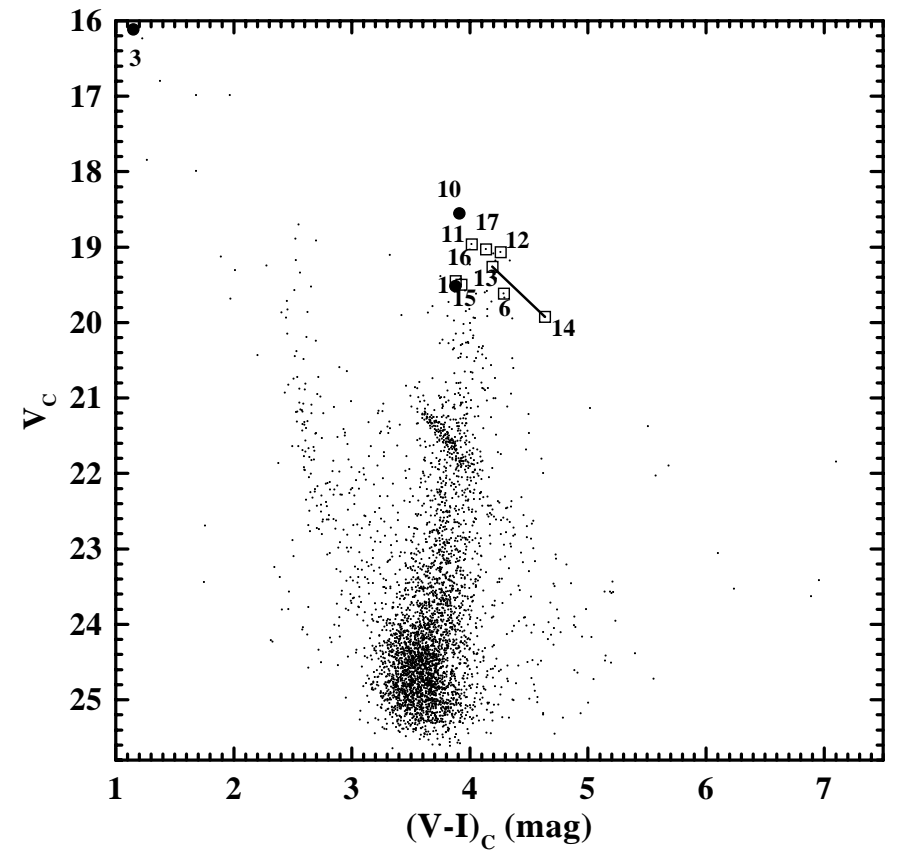

Fig. 2. $V$ vs. $(V-I)$ Colour Magnitude Diagram of Terzan 1 obtained from HST photometry, calibrated to Cousins photometry. Probable members are identified by open squares. Nonmembers are indicated by filled circles. The solid line shows the two more metal-rich stars 13 and 14. Stars 2, 4, 5, 7, 8, 9 are not indicated since they had only Danish photometry (see Sect. 3.2).

Total exposure times of one to two hours were required due to the faint magnitudes of these object $(18<V<22)$, which in addition have an important reddening in the visible. The exposure times were kept to 30-45 min maximum to limit the cosmic ray effects, and series of spectra were added to improve the $S / N$ ratios. The log of observations is shown in Table 2, where columns give respectively: objects denomination according to Figs. 1 and 2, angular distance from the cluster center, integration time and number of individual measurements of each star, epoch of observation and $S / N$ ratios. $S / N$ ratios were estimated in the region $6000-6600 \AA$, over the final sum of spectra for each object.

He-Ar lamps were measured immediately after each object exposure for wavelength calibration. Spectrophotometric standard stars (Taylor 1984; Stone \& Baldwin 1983) were measured each night for flux calibration.

Reductions were carried out using the IRAF package. Cosmic rays were removed and bias subtraction and flatfield correction were made in the standard way. Due to the high density of the field, spectra were extracted taking care to avoid contamination by other stars, which was achieved through a proper definition of apertures. In some cases this leads to some flux loss, decreasing the $S / N$ ratios. Typical errors of the wavelength calibration are $\sim 0.07 \AA$. Flux calibrations, atmospheric extinction corrections and reddening corrections were performed in order to have the correct continuum shape. Final spectra 
Table 1. Instrumental configuration.

\begin{tabular}{lllll}
\hline Obs. Tel. & Spec. & CCD & Grating & $R$ \\
\hline ESO 1.52 m & B\&C & Loral $2048 \times 2048$ & $600 \mathrm{l} / \mathrm{mm}$ & $4 \AA$ \\
LNA1.60 m & B\&C & SITe $1024 \times 1024$ & $600 \mathrm{l} / \mathrm{mm}$ & $5 \AA$ \\
\hline
\end{tabular}

Table 2. Log of observations.

\begin{tabular}{ccccccc}
\hline star & $\begin{array}{c}d \\
\left({ }^{\prime \prime}\right)\end{array}$ & $\begin{array}{c}t_{\text {exp }} \\
(\min )\end{array}$ & $n_{\exp }$ & Date & $S / N$ & site \\
\hline 1 & 48 & 30,40 & 3 & 94 Jun. 14, 15, 16 & 25 & ESO \\
2 & 34 & 40 & 1 & 94 Jun. 15, 17 & 6 & ESO \\
3 & 35 & 30,40 & 3 & 94 Jun. 14, 15, 16 & 40 & ESO \\
4 & 20 & 30 & 2 & 99 Aug. 06 & 18 & LNA \\
& & 30 & 1 & 94 Jun. 17 & 14 & ESO \\
5 & 23 & 30 & 2 & 99 Aug. 03 & 11 & LNA \\
6 & 12 & 30 & 4 & 99 Aug. 05 & 13 & LNA \\
7 & 14 & 45 & 1 & 94 Jun. 17 & 6 & ESO \\
8 & 15 & 30,45 & 2 & 94 Jun. 17 & 10 & ESO \\
9 & 30 & 30 & 3 & 99 Aug. 05 & 15 & LNA \\
10 & 31 & 30 & 4 & 99 Aug. 04 & 15 & LNA \\
11 & 37 & 30 & 4 & 99 Aug. 02 & 12 & LNA \\
12 & 37 & 30 & 4 & 99 Aug. 02 & 15 & LNA \\
13 & 35 & 30 & 4 & 99 Aug. 04 & 11 & LNA \\
14 & 42 & 30 & 4 & 99 Aug. 02 & 8 & LNA \\
15 & 26 & 30 & 4 & 99 Aug. 04 & 9 & LNA \\
16 & 23 & 30 & 4 & 99 Aug. 03 & 10 & LNA \\
17 & 22 & 30 & 4 & 99 Aug. 03 & 13 & LNA \\
\hline
\end{tabular}

were smoothed with a Gaussian filter to reduce some high frequency noise, reducing spectral resolutions to final values of $7 \AA F W H M$ (LNA) and $5 \AA F W H M$ (ESO).

The distribution of the sample stars (Fig. 1), and their angular distances from the center (Table 2), indicate locations well within the half light radius $r_{\mathrm{h}}=229^{\prime \prime}$, and within the radius containing $20 \%$ of the cluster light $r_{20}=81^{\prime \prime}$ (Trager et al. 1995).

\section{Kinematical and atmospheric stellar parameters}

\subsection{Radial velocities}

Radial velocities were derived with the IRAF task fxcor, which uses a cross-correlation Fourier method.

As templates, $12 \mathrm{G}$ and $\mathrm{K}$ giants stars were selected from the Jacoby et al. (1984) catalogue, which have approximately the same spectral resolution $(4.5 \AA F W H M)$ of our spectra. Each template spectrum was also smoothed with the same Gaussian filter used for our program stars to achieve the resolution of $\approx 7 \AA F W H M$ (LNA) or $5 \AA F W H M(\mathrm{ESO})$.

The continua of objects and templates were normalized for the calculation of radial velocities. Different regions in

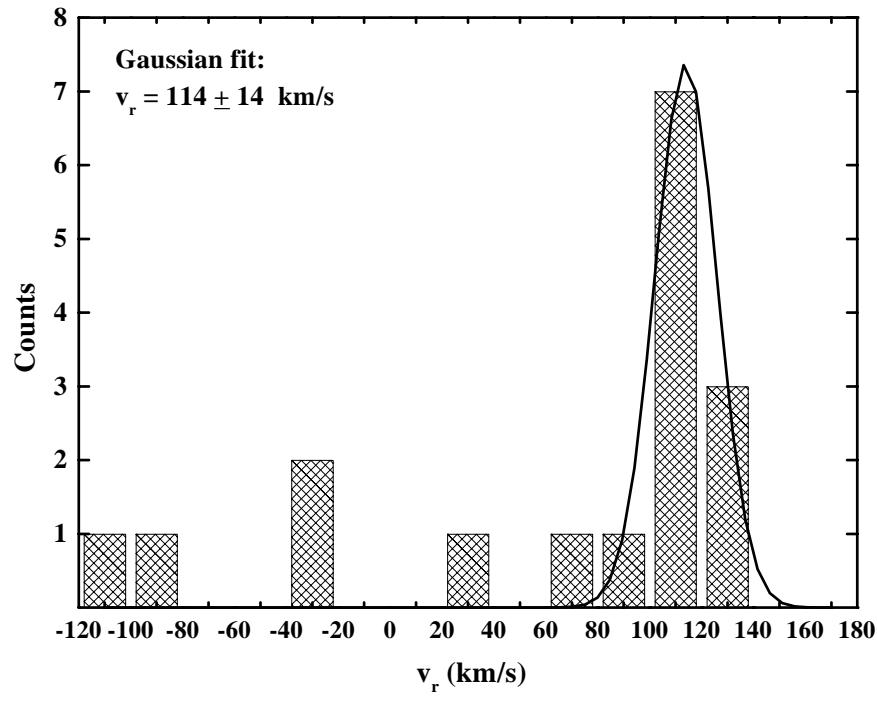

Fig. 3. Heliocentric velocity distribution of program stars. The Gaussian fit shows the probable Terzan 1 members.

Table 3. Heliocentric radial velocities, $V$ and $V-I$ (HST or Danish photometry) and derived effective temperatures.

\begin{tabular}{llccc}
\hline star & $v_{\mathrm{r}} \pm \sigma_{v_{\mathrm{r}}}$ & $V$ & $(V-I)$ & $T_{\text {eff }}$ \\
\hline 1 & $-30 \pm 5$ & 19.52 & 3.88 & $4100^{*}$ \\
2 & $-36 \pm 12$ & 18.77 & 4.25 & 4100 \\
3 & $-111 \pm 12$ & 16.12 & 1.15 & 4500 \\
4 & $+107 \pm 18$ & 18.51 & 4.70 & 3800 \\
& $+137 \pm 26$ & & & \\
5 & $+134 \pm 12$ & 18.66 & 4.64 & 3900 \\
6 & $+110 \pm 26$ & 19.62 & 4.29 & 4300 \\
7 & $-84 \pm 7$ & 19.15 & 3.71 & 5200 \\
8 & $+105 \pm 19$ & 18.58 & 4.74 & 3900 \\
9 & $+121 \pm 16$ & 18.73 & 4.71 & 3900 \\
10 & $+20 \pm 10$ & 18.56 & 3.91 & 5000 \\
11 & $+126 \pm 14$ & 18.96 & 4.02 & 5000 \\
12 & $+93 \pm 21$ & 19.07 & 4.26 & 4300 \\
13 & $+109 \pm 25$ & 19.26 & 4.19 & 4400 \\
14 & $+101 \pm 11$ & 19.93 & 4.64 & 3900 \\
15 & $+101 \pm 13$ & 19.50 & 3.93 & 5000 \\
16 & $+62 \pm 8$ & 19.45 & 3.88 & 5100 \\
17 & $+103 \pm 12$ & 19.03 & 4.14 & 4500 \\
\hline
\end{tabular}

* Effective temperature determination with the code HALO.

the spectra were defined in order to give highest crosscorrelation peaks for each considered template. The final error on the radial velocity for each object is represented by the dispersion of the values obtained from each template for each single star measurement, weighted according to their respective $S / N$ ratios. This gives a more robust estimate of the real errors. Results are shown in Table 3, where radial velocities are corrected to the heliocentric system. 
Figure 3 shows the radial velocity distribution for the observed stars. It can be seen that 11 objects seem to belong to the same stellar system. Stars labeled 1, 2, 3, 7 and 10 clearly do not belong to the globular cluster system and star 16 seems to have a small probability to be a Terzan 1 member. From the 11 probable members we obtain a mean radial velocity of $114 \pm 14 \mathrm{~km} \mathrm{~s}^{-1}$, considerably higher than the value of $v_{\mathrm{r}}=35 \pm 15 \mathrm{~km} \mathrm{~s}^{-1}$ reported by Armandroff \& Zinn (1988). This discrepancy can be expected, since Armandroff \& Zinn (1988) derived the radial velocity from integrated-light spectroscopy, where some non-member stars were possibly included (see Fig. 3).

\subsection{Effective temperatures}

Effective temperatures were estimated using the colourtemperature calibration grids of Houdashelt et al. (2000a,b). A rough estimate of spectral type for each star was first done, in order to select between the calibration grids for $\mathrm{F}-\mathrm{K}$ and $\mathrm{M}$ giants; for both grids we built colourtemperature relations for giant stars $(0 \leq \log g \leq 3)$ of metallicities in range $-3 \leq[\mathrm{Fe} / \mathrm{H}] \leq+0.25$. The derived relations between effective temperature as a function of dereddened $(V-I)_{0}$ colours, $T_{\text {eff }}=f\left[(V-I)_{0}\right]$ have standard deviations varying from $\sigma=50$ to $80 \mathrm{~K}$, depending on the metallicity. Figures $4 \mathrm{a}, \mathrm{b}$ show these relations for $\mathrm{F}-\mathrm{K}$ and $\mathrm{M}$ stars.

The final fitting functions for $\mathrm{F}-\mathrm{K}$ and $\mathrm{M}$ giant stars were obtained by averaging all curves of different metallicities. The following colour-temperature expressions were obtained:

$\mathrm{F}-\mathrm{K}$ giants:

$T_{\text {eff }}=8317-4688 \times(V-I)_{0}+1219 \times(V-I)_{0}^{2}$

$$
0.2 \leq(V-I)_{0} \leq 2.0
$$

\section{$\mathrm{M}$ giants:}

$T_{\text {eff }}=6982-3951 \times(V-I)_{0}+1666 \times(V-I)_{0}^{2}$

$$
-259 \times(V-I)_{0}^{3}
$$

$1.8 \leq(V-I)_{0} \leq 3.4$.

The uncertainties in metallicities lead to maximum errors in $T_{\text {eff }}$ of $\approx \pm 70 \mathrm{~K}$ and $\approx \pm 100 \mathrm{~K}$, respectively for the two expressions above.

As described in Sect. 2, $V-I$ colours were measured from the HST data for most stars; the other stars measured with the Danish telescope were scaled to the zero point derived from HST photometry, tied to the JohnsonCousins system. Stars 4, 5, 8 and 9 resulted to show redder colours relative to the other giants (see Table 3 ), very probably due to crowding; for this reason stars for which only Danish photometry was available were not shown in Fig. 2. Also, the effective temperature values for these stars are consequently underestimated.

In Ortolani et al. (1999) two values of redenning were estimated for Terzan 1, depending on the template used, $E(V-I)=2.70$ and 2.99 , using respectively as templates the metal rich cluster NGC 6553 and the metal poor cluster M30. We found that $T_{\text {eff }}$ estimates using $E(V-I)=2.99$ present lower fitting deviations of the
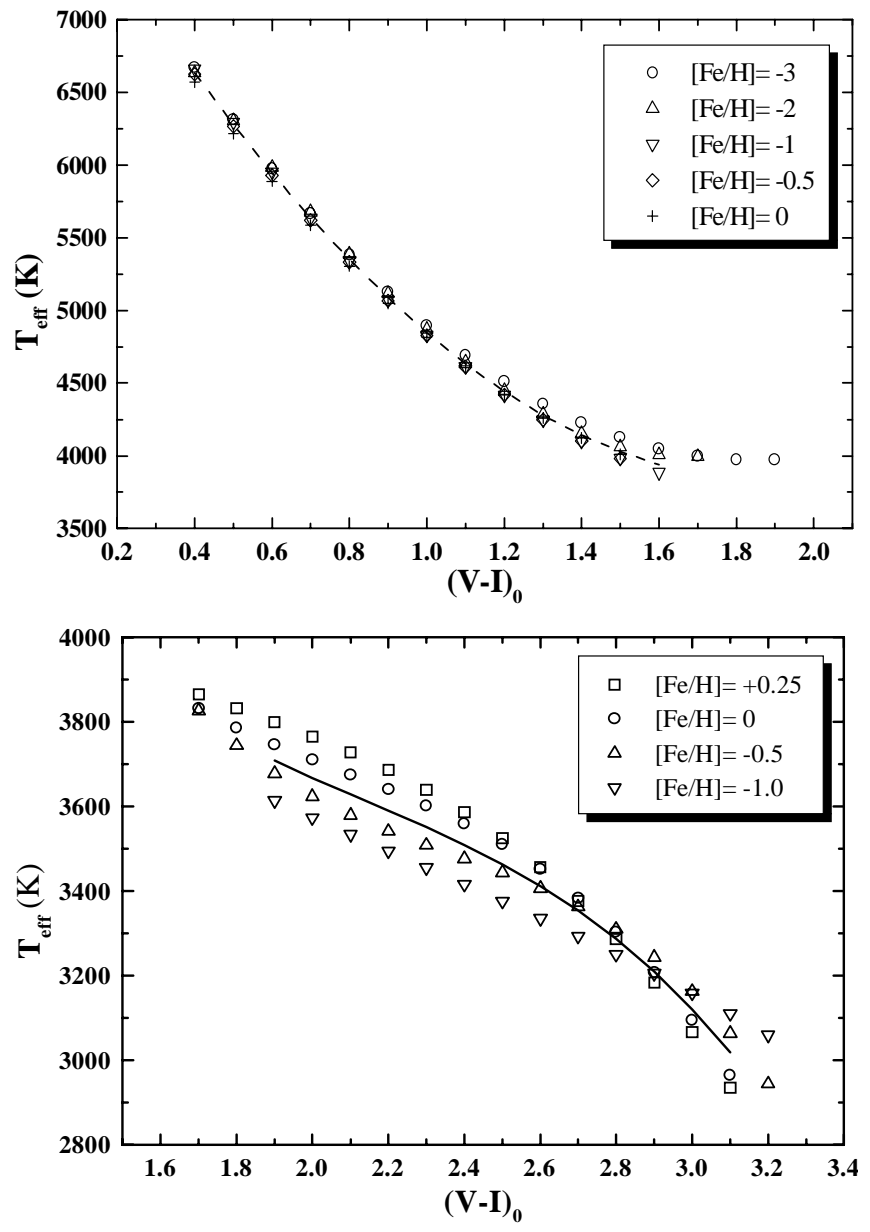

Fig. 4. Metallicity dependence of effective temperature versus $V-I$ for $\mathrm{F}-\mathrm{K}$ (upper panel) and M (lower panel) giant stars, based on models by Houdalshelt et al. (2000a,b).

$[\mathrm{Fe} / \mathrm{H}]$ estimates (see following section), and they are more compatible with intensities of molecular bands. In Table 3 we show the observed visual magnitudes, colour indices $V-I$ and effective temperatures estimated using $E(V-I)=2.99$.

\subsection{Metallicity}

The code HALO (Cayrel et al. 1991; Barbuy et al. 2001) allows us to estimate the atmospheric parameters $\left(T_{\text {eff }}\right.$, $\log g,[\mathrm{Fe} / \mathrm{H}])$ of an observed star by interpolation on a grid of synthetic spectra. The grid used here contains 2522 stellar synthetic spectra computed as described in Barbuy et al. (2001), in the wavelength region $\lambda \lambda 4600$ $5600 \AA$, with atmospheric parameters in the intervals: $4000<T_{\text {eff }}<7000 \mathrm{~K}, 0<\log g<5$ and $-3<[\mathrm{Fe} / \mathrm{H}]<$ +0.3 dex and $[\mathrm{Mg} / \mathrm{Fe}]=0.0,+0.4$. The fitting procedure and photospheric models used in the code HALO are described in detail in Cayrel et al. (1991) and Barbuy et al. (2001).

The relatively low $S / N$ ratios of our observed spectra do not allow us to calculate the three atmospheric parameters using HALO for most of the spectra. Only for stars 1 
and 3 , with a higher $S / N$, it was possible to estimate effective temperatures and metallicities using HALO. For the other stars we assumed $T_{\text {eff }}$ values estimated from the $V-I$ photometry (see Sect. 3.2). We derived $[\mathrm{Fe} / \mathrm{H}]$ for a given $T_{\text {eff }}$, combined to a set of $\log g$ values: $0.5,1,1.5$ and 2 . The procedure used to estimate metallicities of low $S / N$ ratio spectra was:

a) different regions of each spectrum were weighted according to their relative $S / N$ ratios;

b) for a given $T_{\text {eff }}$ and a set of $\log g$ values, we ran HALO for 363 synthetic spectra of different $[\mathrm{Fe} / \mathrm{H}]$ values and $[\mathrm{Mg} / \mathrm{Fe}]$, combined 2 by 2 . A series of $[\mathrm{Fe} / \mathrm{H}]$ values are obtained, which are a mean of the solar and non-solar $[\mathrm{Mg} / \mathrm{Fe}]$ results;

c) for a given $\log g$ and $T_{\text {eff }}$, a final $[\mathrm{Fe} / \mathrm{H}]$ value was adopted, which corresponds to the average of the Gaussian distribution of $[\mathrm{Fe} / \mathrm{H}]$ values (see example in Fig. A.2);

d) the final solution is a weighted average of the solutions obtained for $0.5<\log g<2$, for a given temperature. For the cooler stars of temperatures $T_{\text {eff }}<4500 \mathrm{~K}$, the range $0.5<\log g<1.5$ was considered. The weights are the standard deviations of the Gaussian fits obtained in c). Errors were estimated by taking the weighted average among the solutions of different $\log g$. These errors should be seen as deviations of possible solutions presented by the code HALO which in turn are a function of $S / N$ ratios of the observed spectra. The resulting $[\mathrm{Fe} / \mathrm{H}]$ are given in Table 4. An example of the reliability of this procedure can be seen in Appendix A for star 3. In Appendix B the solutions for each $\log g$ and $T_{\text {eff }}$ for the sample stars are shown.

\section{4. $\alpha$-elements-to-iron ratio}

It is well-known that the $[\alpha$-elements $/ \mathrm{Fe}]$ value for stars gives a direct insight about the chemical enrichment process of a stellar system. Previous work pointed out that the galactic bulge is metal-rich (McWilliam \& Rich 1994) with non-solar values for some $\alpha$-elements relative to $\mathrm{Fe}$, as also found by Idiart et al. (1996), Sadler et al. (1996) and Barbuy et al. (1999). In principle, this indicates an old age and a rapid enrichment of the bulge.

In this work we measured the index $\mathrm{Mg}_{2}$, aiming to obtain $[\mathrm{Mg} / \mathrm{Fe}]$ ratios for each star. The resulting $\mathrm{Mg}_{2}$ indices transformed to the Lick system (Faber et al. 1985) are shown in Table 4 . We calculate an empirical formula $[\mathrm{Mg} / \mathrm{Fe}]=f\left[T_{\text {eff }}, \mathrm{Mg}_{2},[\mathrm{Fe} / \mathrm{H}]\right]$ valid for giant stars $(0.5<\log g<2)$, using stellar data of Borges et al. (1995). For 22 cool giant stars, with $-2.45 \leq[\mathrm{Fe} / \mathrm{H}] \leq+0.25$, $-0.05 \leq[\mathrm{Mg} / \mathrm{Fe}] \leq+0.6$ and $0.024 \leq \mathrm{Mg}_{2} \leq 0.382$, we obtained: $[\mathrm{Mg} / \mathrm{Fe}]=-0.1163+7.26824 \times \mathrm{Mg}_{2}-$ $0.23185 \times[\mathrm{Fe} / \mathrm{H}]-5.78524 \times \theta_{\text {eff }} \times \mathrm{Mg}_{2}$. The fitting $\mathrm{rms}$ is 0.15 dex. Results are shown in Table 4 , with their corresponding uncertainties, when possible. These uncertainties were estimated by obtaining the rms deviation of the values measured for each independent spectrum, weighted

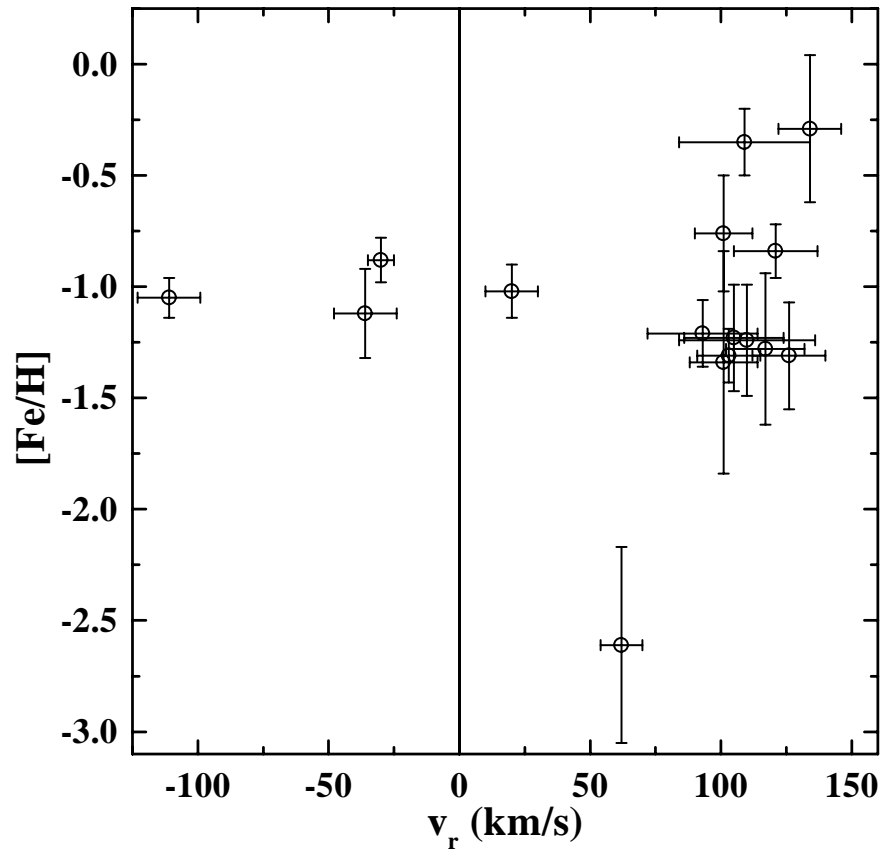

Fig. 5. Metallicity $[\mathrm{Fe} / \mathrm{H}]$ vs. radial velocity $v_{\mathrm{r}}\left(\mathrm{km} \mathrm{s}^{-1}\right)$ for sample stars.

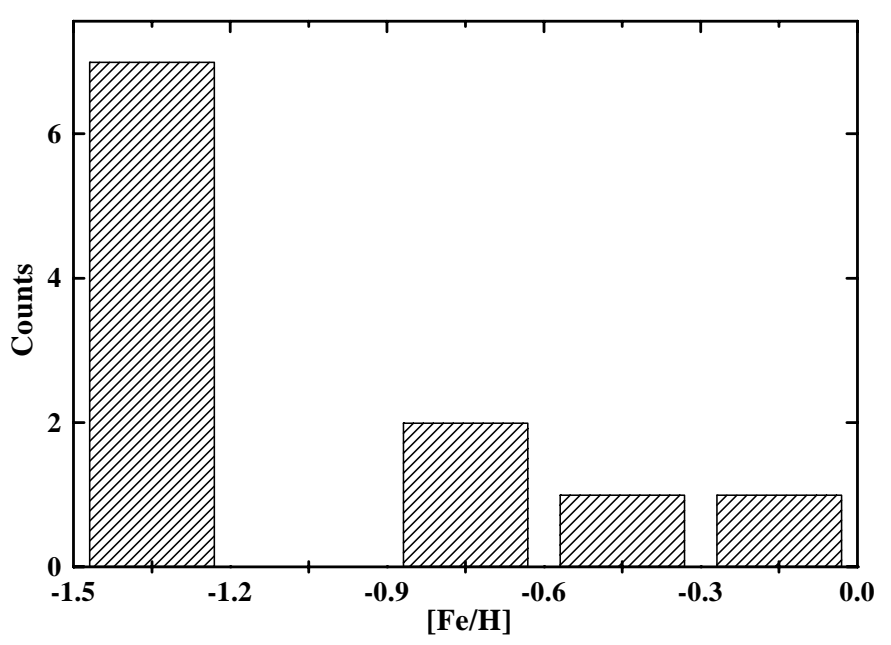

Fig. 6. Histogram of metallicity distribution of Terzan 1 probable members.

by its $S / N$. For cases where $\mathrm{Mg}_{2}$ measures were derived from one spectrum, rms values are not given.

For a given $\mathrm{Mg}_{2}$ and $[\mathrm{Fe} / \mathrm{H}]$, an uncertainty of $\pm 200 \mathrm{~K}$ in temperature gives mean errors of \pm 0.07 dex in $[\mathrm{Mg} / \mathrm{Fe}]$. Likewise, errors of $20 \%$ in $\mathrm{Mg}_{2}$, give errors of 0.01 to $0.03 \mathrm{dex}$ in $[\mathrm{Mg} / \mathrm{Fe}]$, and errors of $0.3 \mathrm{dex}$ in $[\mathrm{Fe} / \mathrm{H}]$, give errors of 0.07 dex in $[\mathrm{Mg} / \mathrm{Fe}]$.

\subsection{Discussion}

Figures 5 and 6 show the metallicity distribution of Terzan 1 probable members, according to their radial velocities. It can be seen that most stars lie on the metalpoor side of the distribution, displaying an average value $[\mathrm{Fe} / \mathrm{H}]=-1.27 \pm 0.05$. Star 16 can be discarded, since it 
Table 4. Metallicities $[\mathrm{Fe} / \mathrm{H}]$ derived with the code $\mathrm{HALO}$ (Sect. 3.3) and $\mathrm{Mg}_{2}$ index and $[\mathrm{Mg} / \mathrm{Fe}]$ values (Sect. 3.4).

\begin{tabular}{lcccccc}
\hline star & {$[\mathrm{Fe} / \mathrm{H}]$} & $\sigma_{[\mathrm{Fe} / \mathrm{H}]}$ & $\mathrm{Mg}_{2}$ & $\sigma_{\mathrm{Mg}_{2}}$ & {$[\mathrm{Mg} / \mathrm{Fe}]$} & Comments \\
\hline 1 & -0.88 & 0.1 & 0.39 & 0.14 & +0.14 & non-member \\
2 & -1.12 & 0.2 & 0.27 & $:$ & +0.18 & non-member, Danish photometry \\
3 & -1.05 & 0.09 & 0.14 & 0.05 & +0.25 & non-member \\
4 & -1.28 & 0.34 & 0.24 & 0.03 & +0.1 & Danish photometry \\
5 & -0.29 & 0.33 & 0.55 & 0.16 & -0.24 & Danish photometry \\
6 & -1.24 & 0.25 & 0.30 & 0.04 & +0.31 & \\
7 & & & -0.02 & $:$ & & non-member, Danish photometry \\
8 & -1.23 & 0.24 & 0.58 & 0.20 & -0.06 & Danish photometry \\
9 & -0.84 & 0.12 & 0.23 & 0.06 & -0.01 & \\
10 & -1.02 & 0.12 & 0.17 & 0.02 & +0.38 & \\
11 & -1.31 & 0.24 & 0.26 & $:$ & +0.49 & \\
12 & -1.21 & 0.15 & 0.36 & $:$ & +0.35 & \\
13 & -0.35 & 0.15 & 0.35 & $:$ & +0.45 & \\
14 & -0.76 & 0.26 & 0.25 & 0.05 & +0.01 & \\
15 & -1.34 & 0.50 & 0.035 & $:$ & +0.22 & \\
16 & -2.61 & 0.44 & 0.24 & 0.08 & +0.86 & \\
17 & -1.31 & 0.12 & 0.21 & 0.02 & +0.36 & \\
\hline
\end{tabular}

has a marginal probability of belonging to Terzan 1 (as discussed in Sect. 3.1) and additionally it seems to have a very low $[\mathrm{Fe} / \mathrm{H}]$ value. Possibly it is a halo object.

Star 14 is the only object that seems to belong to the cool red giant branch, typical of metal rich clusters. The $[\mathrm{Fe} / \mathrm{H}]$ value found $([\mathrm{Fe} / \mathrm{H}]=-0.76 \pm 0.26)$ is closer to star $9([\mathrm{Fe} / \mathrm{H}]=-0.84 \pm 0.12)$ and lower than that of the two other metal-rich stars $(-0.39$ and -0.29 dex $)$. Its solar $[\mathrm{Mg} / \mathrm{Fe}]$, similar to stars 5 and 9 , is different from that of star 13 with $[\mathrm{Mg} / \mathrm{Fe}] \approx+0.5$. Stars 5,9 and 14 could be field disk stars. On the other hand, for stars 5 and 9 the derived $T_{\text {eff }}$ value from $V-I$ Danish photometry probably gives an underestimated temperature, as discussed in Sect. 3.2, which can lead to an uncertain $[\mathrm{Mg} / \mathrm{Fe}]$. Star 13 has abundance values compatible with $[\mathrm{Fe} / \mathrm{H}]$ and $[\alpha-$ elements $/ \mathrm{Fe}]$ ratios of bulge stars as pointed out in Sect. 3.4. In Fig. 2 the two more metal-rich stars (13 and 14) are shown by a solid line. The most metal-poor star (star 16) has non-solar $[\mathrm{Mg} / \mathrm{Fe}]$ value, compatible with being a halo star.

The stars with $[\mathrm{Fe} / \mathrm{H}] \approx-1.3$ are probable members (stars $4,6,8,11,12,15,17$ ) and present non-solar $[\mathrm{Mg} / \mathrm{Fe}]$ ratios. The other more metal-rich stars (stars $5,9,13,14$ ), with the same radial velocities as the probable members, can be field disk or bulge stars. The present results shown in Figs. 5 and 6 favour the metal-poor interpretation combined with the second parameter effect, as concluded by Ortolani et al. (1999).

The presence of the few more metal-rich stars could be explained simply by having interloping bulge stars. Minniti (1996) presented velocities and metallicities for bulge field stars along a line of sight close to this one: his Fig. 10 shows that field stars with $\sim 120 \mathrm{~km} \mathrm{~s}^{-1}$ and
$[\mathrm{Fe} / \mathrm{H}] \sim-0.5$ are rather common in this direction. Another interpretation that would need more extensive data to be checked is a scenario of capture of field stars, by an initially metal poor massive cluster, which during a Hubble time might change its stellar content. Such possibility was discussed for the bulge cluster HP 1 (Bica et al. 1997). The simulations suggest that captures are efficient for cluster masses $\approx 10^{5} M_{\odot}$ or larger, and streaming velocities around $50 \mathrm{~km} \mathrm{~s}^{-1}$. Assuming an integrated magnitude $V=15.9$ (Harris 1996), a reddening $E(B-V)=2.48$ and a distance from the sun $d_{\odot}=5.2 \mathrm{kpc}$ (Ortolani et al. 1999), the absolute magnitude for Terzan 1 is $M_{V}=-5.4$. This updated value is similar to those of other central bulge globular clusters (Harris 1996), implying in a cluster mass of $\approx 10^{4} M_{\odot}$. Considering evaporation and discshocking effects, bulge clusters are expected to have been more massive in the past. The radial velocity of Terzan 1 (Sect. 3.1) suggests a rather high streaming velocity in the bulge for captures, at its current position in the orbit. Although the current mass and streaming velocity of Terzan 1 do not appear particularly favourable for capture of bulge stars, it is possible that this mechanism operated efficiently over a significant fraction of the cluster lifetime.

\section{Concluding remarks}

In this work intermediate resolution spectra of 17 stars in the field of Terzan 1 were measured, and 11 of them were found to be probable members of the cluster. For each star, atmospheric parameters $T_{\text {eff }},[\mathrm{Fe} / \mathrm{H}]$ and $[\mathrm{Mg} / \mathrm{Fe}]$ were derived. We obtained a dominant metal-poor $[\mathrm{Fe} / \mathrm{H}]$ of $\approx-1.3$ component. Most probable members stars have 


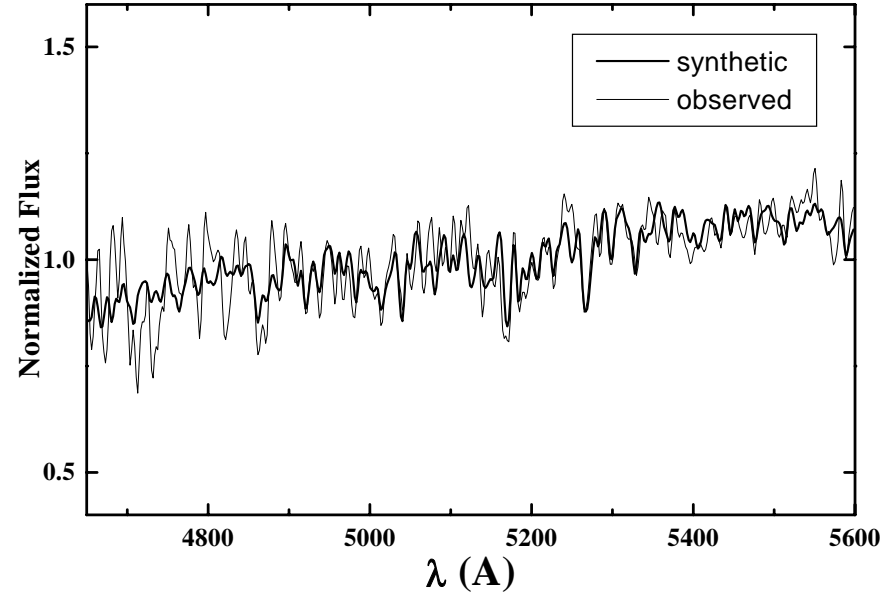

Fig. A.1. Observed spectrum of star 3 (thin line) and synthetic spectrum (thick line) computed with $T_{\text {eff }}=4600 \mathrm{~K}, \log g=1.0$ and $[\mathrm{Fe} / \mathrm{H}]=-1.10$.

Table A.1. Atmospheric parameters results for star 3 .

\begin{tabular}{lccc}
\hline mode & $T_{\text {eff }}$ & $\log g$ & {$[\mathrm{Fe} / \mathrm{H}]$} \\
\hline 3 free parameters & $4600 \pm 200$ & $1.10 \pm 0.18$ & $-1.15 \pm 0.15$ \\
$\log g$ fixed & $4500 \pm 50$ & $0.5,1,1.5,2$ & $-1.09 \pm 0.18$ \\
$T_{\text {eff fixed }}$ and $\log g$ fixed & 4500 & $1.10 \pm 0.10$ & $-1.18 \pm 0.17$ \\
$T_{\text {eff }}$ and & & $0.5,1,1.5,2$ & $-1.11 \pm 0.11$ \\
\hline
\end{tabular}

Table A.2. Resulting atmospheric parameters for different $\mathrm{S} / \mathrm{N}$ of star 3 .

\begin{tabular}{cc}
\hline$S / N$ & {$[\mathrm{Fe} / \mathrm{H}]$} \\
\hline 40 & $-1.05 \pm 0.09$ \\
12 & $-1.22 \pm 0.16$ \\
6 & $-1.38 \pm 0.18$ \\
\hline
\end{tabular}

non-solar $[\mathrm{Mg} / \mathrm{Fe}]$, except for star 14 which belongs to the cool red giant branch and presents higher metallicity.

The present results confirm that Terzan 1 is a metalpoor second parameter globular cluster.

Acknowledgements. TI acknowledges a Fapesp pos-doc fellowship No 97/13083-7. BB and EB acknowledge partial financial support from CNPq and Fapesp.

\section{Appendix A: Metallicity determination with the code HALO}

Stellar atmospheric parameters of star 3, for which a spectrum of $S / N=40$ is available, were estimated with the code HALO by using different reference synthetic spectra, in order to check the reliability of the procedure of metallicity determination described in Sect. 3.3. This check can be seen as an convergence test of the code HALO relative to the several solutions resulting from the different combinations of synthetic spectra.
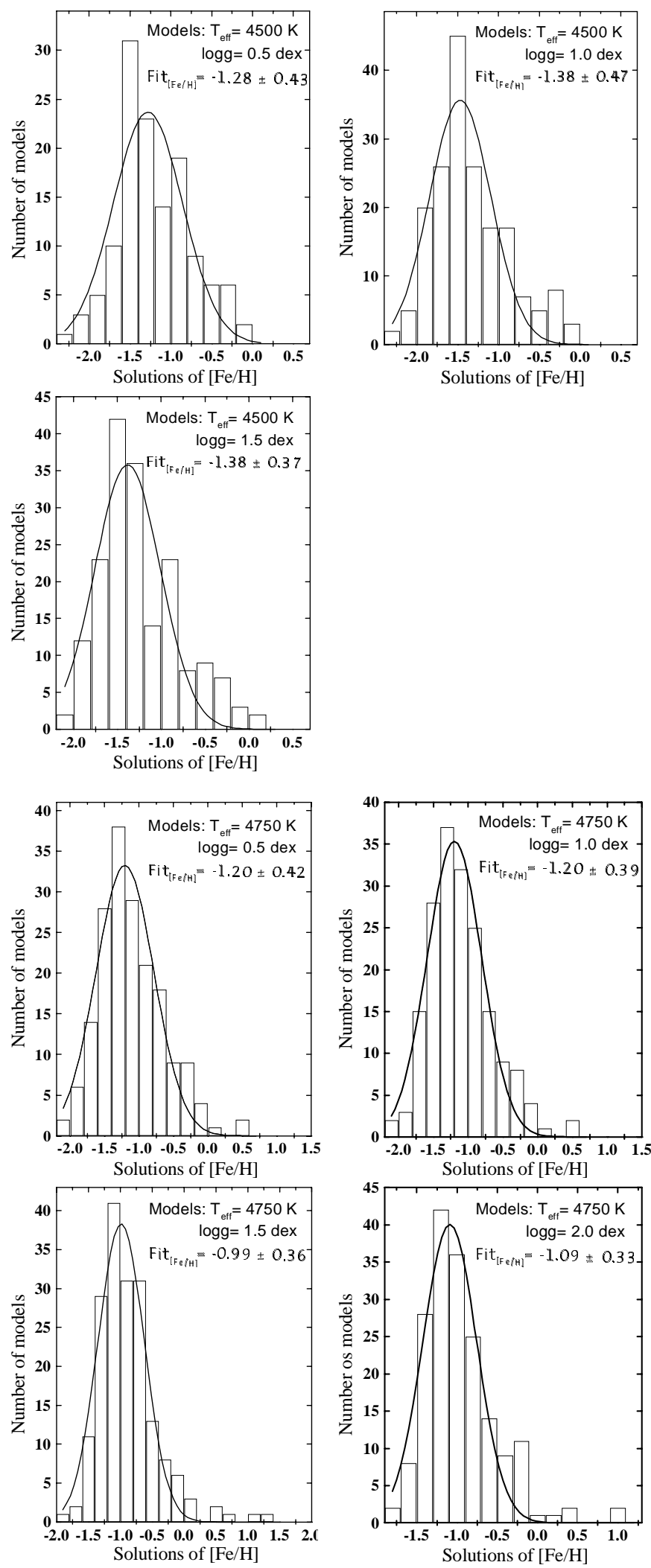

Fig. A.2. Histograms of possible $[\mathrm{Fe} / \mathrm{H}]$ solutions for star 3 , using spectrum of $S / N=12$. [Fe/H] values are estimated using a grid of synthetic spectra of stellar parameters of $T_{\text {eff }}=4500$, $4750 \mathrm{~K}, \log g=0.5,1.0,1.5,2.0$ and a range of metallicities $-3<[\mathrm{Fe} / \mathrm{H}]<+0.3$. The final weighted average is $-1.26 \pm$ 0.18 . 
Table B.1. Partial solutions of $[\mathrm{Fe} / \mathrm{H}]$ as a function of $\log g$ and $T_{\text {eff }}$.

\begin{tabular}{|c|c|c|c|c|c|}
\hline Star & $T_{\text {eff }}$ & $\log g=0.5$ & $\log g=1.0$ & $\log g=1.5$ & $\log g=2$ \\
\hline \multirow[t]{2}{*}{2} & 4000 & $-0.84 \pm 0.44$ & $-1.08 \pm 0.65$ & $-1.90 \pm 0.97 *$ & \\
\hline & 4250 & $-0.84 \pm 0.33$ & $-1.57 \pm 0.50$ & $-1.69 \pm 0.49$ & \\
\hline 4 & 4000 & $-1.02 \pm 0.54$ & $-1.08 \pm 0.55$ & $-1.74 \pm 0.55$ & \\
\hline 5 & 4000 & $-0.13 \pm 0.90 *$ & $-0.20 \pm 0.50$ & $-0.36 \pm 0.43$ & \\
\hline \multirow[t]{2}{*}{6} & 4000 & $-1.10 \pm 0.46$ & $-1.34 \pm 0.46$ & $-2.04 \pm 0.81 *$ & \\
\hline & 4250 & $-1.27 \pm 0.40$ & $-1.98 \pm 0.76 *$ & $-2.01 \pm 0.63 *$ & \\
\hline 8 & 4000 & $-0.81 \pm 0.42$ & $-0.98 \pm 0.43$ & $-1.89 \pm 0.42$ & \\
\hline \multirow[t]{2}{*}{9} & 4000 & $-0.79 \pm 0.34$ & $-0.99 \pm 0.50$ & $-1.72 \pm 0.78 *$ & \\
\hline & 4250 & $-0.72 \pm 0.15$ & $-1.20 \pm 0.42$ & $-1.41 \pm 0.40$ & \\
\hline \multirow[t]{3}{*}{10} & 4750 & $-1.24 \pm 0.48$ & $-1.28 \pm 0.48$ & $-1.07 \pm 0.48$ & $-1.15 \pm 0.45$ \\
\hline & 5000 & $-1.19 \pm 0.39$ & $-1.23 \pm 0.43$ & $-0.94 \pm 0.41$ & $-0.88 \pm 0.42$ \\
\hline & 5250 & $-1.02 \pm 0.31$ & $-1.00 \pm 0.31$ & $-0.72 \pm 0.44$ & $-0.74 \pm 0.43$ \\
\hline \multirow[t]{3}{*}{11} & 4750 & $-1.62 \pm 0.65 *$ & $-1.62 \pm 0.65 *$ & $-1.43 \pm 0.57$ & $-1.43 \pm 0.56$ \\
\hline & 5000 & $-1.59 \pm 0.63 *$ & $-1.66 \pm 0.61 *$ & $-1.32 \pm 0.50$ & $-1.29 \pm 0.53$ \\
\hline & 5250 & $-1.51 \pm 0.59 *$ & $-1.57 \pm 0.60 *$ & $-1.28 \pm 0.54$ & $-1.23 \pm 0.52$ \\
\hline \multirow[t]{2}{*}{12} & 4250 & $-1.15 \pm 0.42$ & $-1.19 \pm 0.42$ & $-1.25 \pm 0.31$ & \\
\hline & 4500 & $-1.35 \pm 0.30$ & $-1.00 \pm 0.41$ & $-1.15 \pm 0.40$ & \\
\hline \multirow[t]{2}{*}{13} & 4250 & $-0.36 \pm 0.17$ & $-0.56 \pm 0.29$ & $-0.57 \pm 0.38$ & \\
\hline & 4500 & $-0.30 \pm 0.22$ & $-0.28 \pm 0.23$ & $-0.03 \pm 0.36$ & \\
\hline \multirow[t]{2}{*}{14} & 4000 & $-0.78 \pm 0.54$ & $-0.97 \pm 0.56$ & $-1.83 \pm 1.03 *$ & \\
\hline & 4250 & $-0.58 \pm 0.37$ & $-0.95 \pm 0.48$ & $-0.77 \pm 0.58$ & \\
\hline \multirow[t]{3}{*}{15} & 4750 & $-1.76 \pm 0.69$ & $-1.65 \pm 0.69$ & $-1.20 \pm 0.55$ & $-1.07 \pm 0.60$ \\
\hline & 5000 & $-2.01 \pm 0.79 *$ & $-1.92 \pm 0.89 *$ & $-1.33 \pm 0.58$ & $-1.08 \pm 0.56$ \\
\hline & 5250 & $-2.30 \pm 0.94 *$ & $-2.12 \pm 0.93 *$ & $-1.69 \pm 0.68$ & $-1.31 \pm 0.53$ \\
\hline \multirow[t]{2}{*}{16} & 5000 & $-2.76 \pm 1.31$ & $-2.77 \pm 1.22$ & $-2.39 \pm 1.16$ & $-2.06 \pm 0.98$ \\
\hline & 5250 & $-3.17 \pm 1.39$ & $-3.01 \pm 1.36$ & $-2.84 \pm 1.38$ & $-2.57 \pm 1.27$ \\
\hline \multirow[t]{3}{*}{17} & 4250 & $-0.93 \pm 0.38$ & $-1.75 \pm 0.52$ & $-1.83 \pm 0.39$ & \\
\hline & 4500 & $-1.31 \pm 0.44$ & $-1.52 \pm 0.42$ & $-1.36 \pm 0.31$ & \\
\hline & 4750 & $-1.31 \pm 0.46$ & $-1.36 \pm 0.44$ & $-1.10 \pm 0.30$ & $-1.08 \pm 0.35$ \\
\hline
\end{tabular}

Note: $*[\mathrm{Fe} / \mathrm{H}]$ solution not considered in the final average.

Firstly, we used a set of 48 synthetic spectra for a simultaneous estimate of $T_{\text {eff }}, \log g$ and $[\mathrm{Fe} / \mathrm{H}]$ in the intervals: $4250<T_{\text {eff }}<4750 \mathrm{~K}, 1<\log g<3,0<[\mathrm{Fe} / \mathrm{H}]<$ -1.5 , which allows to have 9 combinations of 4 models for each calculation. One of the best $T_{\text {eff }}, \log g$ and $[\mathrm{Fe} / \mathrm{H}]$ fits is shown in Fig. A.1.

Secondly, $T_{\text {eff }}$ and $[\mathrm{Fe} / \mathrm{H}]$ were taken as free parameters, and their values were estimated relative to the $\log g$ values $=0.5,1,1.5$ and 2 , and an average of the results for each $\log g$ is derived. In the same way, we assumed $T_{\text {eff }}$ values, with $\log g$ and $[\mathrm{Fe} / \mathrm{H}]$ as free parameters. It is important to emphasize that these two preceding procedures work only if the observed spectra have a reasonable $S / N$ ratio, since for a worse $S / N$ the solutions for three or two free parameters may not converge.

Finally we calculate $[\mathrm{Fe} / \mathrm{H}]$ according to the procedure described in Sect. 3.3. Table A.1 shows the resulting atmospheric parameters. It can be noted that the solutions converge well, no matter which procedure is used.

The dependence of the results on $S / N$ was also checked. Using the IRAF task "mknoise", two different intensities of noise for the spectrum of star 3 were simulated, in order to see the resulting $[\mathrm{Fe} / \mathrm{H}]$ solutions, shown in Table A.2. Lower $S / N$ ratios tend to decrease the final
$[\mathrm{Fe} / \mathrm{H}]$ values by up to $\approx 0.23 \operatorname{dex}($ for $S / N=6$ ), but at the same time they increase the standard deviations of possible solutions. Therefore, the solutions from low $S / N$ spectra are reasonable within the standard deviations derived. Fig. A.2 shows the histograms of the $[\mathrm{Fe} / \mathrm{H}]$ solutions obtained for $0<\log g<2$ and $T_{\text {eff }}=4500$ and $4750 \mathrm{~K}$, for the spectrum of star 3 with $S / N=12$.

\section{Appendix B: Dependence of $[\mathrm{Fe} / \mathrm{H}]$ solutions on $\log g$}

Table B.1 shows the metallicities estimated for different $T_{\text {eff }}$ and $\log g$ values. Note that HALO was ran for a set of models using two or three different effective temperatures, chosen to be as close as possible to the value estimated from the $V-I$ colours.

A striking dependence of $[\mathrm{Fe} / \mathrm{H}]$ on $\log g$ is seen for the cooler stars. For these stars the mean metallicity adopted was an average of the values for $\log g=0.5,1.0$ and 1.5 only, since they are at the tip of the RGB. For the stars with temperatures around $5000 \mathrm{~K}$ the dependence on $\log g$ is much less strong. 


\section{References}

Armandroff, T. E., \& Zinn, R. 1988, AJ, 96, 92

Barbuy, B., Bica, E., \& Ortolani, S. 1998, A\&A, 333, 117

Barbuy, B., Renzini A., Ortolani S., Bica, E., \& Guarnieri, M. D. 1999, A\&A, 341, 539

Barbuy, B., Perrin, M.-N., Katz, D., et al. 2001, A\&A, submitted

Bica, E., Dottori, H., Rodrigues, I. O. F., Ortolani, S., \& Barbuy, B. 1997, ApJ, 482, L49

Borges, A. C., Idiart, T. P., de Freitas Pacheco, J. A., \& Thévenin, F. 1995, AJ, 110, 2408

Faber, S. M., Friel, E., Burstein, D., \& Gaskell, C. M. 1985, ApJS, 57, 711

Fusi Pecci, F. 1997, AJ, 113, 706

Cayrel, R., Perrin, M.-N., Barbuy, B., \& Buser, R. 1991, A\&A, 247, 108

Côté, P. 1999, AJ, 118, 406

Harris, W. E. 1996, AJ, 112, 1487
Houdashelt, M. L., Bell, R. A., Sweigart, A. V., \& Wing, R. F. 2000a, AJ, 119, 1424

Houdashelt, M. L., Bell, R. A., \& Sweigart, A. V. 2000b, AJ, 119,1448

Idiart, T. P., de Freitas Pacheco, J. A., \& Costa, R. D. D. 1996, AJ, 111, 1169

Jacoby, G. H., Hunter, D. A., \& Christian, C. A. 1984, ApJS, 56,257

McWilliam, A., \& Rich, M. 1994, ApJS, 91, 749

Minniti, D. 1995, AJ, 109, 1663

Minniti, D. 1996, ApJ, 459, 579

Ortolani, S., Bica, E., \& Barbuy, B. 1993, A\&A, 267, 66

Ortolani, S., Renzini, A., Gilmozzi, R., et al. 1995, Nature, 377 , 701

Ortolani, S., Barbuy, B., Bica, E., et al. 1999, A\&A, 350, 840

Sadler, E., Terndrup, D., \& Rich, R. M. 1996, AJ, 112, 171

Stone, R. P. S., \& Baldwin, J. A. 1983, MNRAS, 204, 347

Taylor, B. J. 1984, ApJS, 54, 259

Trager, S. C., King, I. R., \& Djorgovski, S. 1995, AJ, 109, 218 